\title{
Additional evidence on the efficacy of different Akirin vaccines assessed on Anopheles arabiensis (Diptera: Culicidae)
}

\author{
Blaženka D. Letinić ${ }^{1,2}$, Marinela Contreras ${ }^{3,4}$, Yael Dahan-Moss ${ }^{1,2}$, Ingrid Linnekugel ${ }^{5}$, José de la Fuente ${ }^{3,6}$ and
} Lizette L. Koekemoer ${ }^{1,2^{*}}$ (D)

\begin{abstract}
Background: Anopheles arabiensis is an opportunistic malaria vector that rests and feeds outdoors, circumventing current indoor vector control methods. Furthermore, this vector will readily feed on both animals and humans. Targeting this vector while feeding on animals can provide an additional intervention for the current vector control activities. Previous results have displayed the efficacy of using Subolesin/Akirin ortholog vaccines for the control of multiple ectoparasite infestations. This made Akirin a potential antigen for vaccine development against An. arabiensis.

Methods: The efficacy of three antigens, namely recombinant Akirin from An. arabiensis, recombinant Akirin from Aedes albopictus, and recombinant Q38 (Akirin/Subolesin chimera) were evaluated as novel interventions for An. arabiensis vector control. Immunisation trials were conducted based on the concept that mosquitoes feeding on vaccinated balb/c mice would ingest antibodies specific to the target antigen. The antibodies would interact with the target antigen in the arthropod vector, subsequently disrupting its function.

Results: All three antigens successfully reduced An. arabiensis survival and reproductive capacities, with a vaccine efficacy of $68-73 \%$.

Conclusions: These results were the first to show that hosts vaccinated with recombinant Akirin vaccines could develop a protective response against this outdoor malaria transmission vector, thus providing a step towards the development of a novel intervention for An. arabiensis vector control.
\end{abstract}

Graphic Abstract: Keywords: Malaria, Vector control, Akirin, Subolesin, Immunisation, Recombinant proteins

\section{Background}

Traditional control methods for arthropod vectors are mainly based on the use of chemical insecticides [1]. Insecticide resistance, due to increased selection pressure, has necessitated the development of new classes of insecticides or other novel control interventions. Novel approaches that exploit the natural behaviour of arthropod vectors can be used to target these ectoparasites. The

*Correspondence: lizette.koekemoer@wits.ac.za

${ }^{1}$ Wits Research Institute for Malaria, School of Pathology, Faculty of Health Sciences, University of the Witwatersrand, Johannesburg, South Africa

Full list of author information is available at the end of the article opportunistic African malaria vector, Anopheles arabiensis, will readily feed on both animals and humans [2]. Targeting this vector while feeding on animals can provide an additional intervention for the current vector control strategies.

Agricultural animals are regularly vaccinated with recombinant vaccines for the control of multiple endoand ectoparasitic infestations [3-5]. These recombinant vaccines contain protective antigens that develop antigen-specific antibodies in the immunised hosts [6]. Arthropod vectors ingest these antibodies when feeding on the vaccinated hosts. The ingested antibodies interact 
with and disrupt the function of the target antigen in the arthropod vectors. This results in physiological changes that affect the vector's biology $[7,8]$. This concept was first demonstrated when cattle were vaccinated with crude extracts from Dermacentor andersoni. Consequently, these ectoparasites were adversely affected when feeding on the vaccinated hosts [9].

The first arthropod vaccines were manufactured and distributed after the discovery of the gut antigen, BM86, in Rhipicephalus microplus $[3,6]$. Gavac ${ }^{\mathrm{TM}}$ and TickGARD ${ }^{\text {PLUS }}$ were used to control tick infestations through cattle immunisation $[3,6,10-13]$. Thereafter, it was proposed that multi-target vaccines directed at the control of several vector species could be developed using evolutionary conserved protective antigens [14]. However, the limiting step in the development of such vaccines is the identification of these antigens [15].

Using expression library immunisation, an evolutionary conserved protective antigen, Subolesin, was discovered in Ixodes scapularis [16-18]. Immunisation of cattle, using recombinant Subolesin, caused several deleterious effects in engorged ticks. This included diminished vectorial capacity, reduced oviposition, and reduced vector survivorship $[17,19]$. The feeding capabilities of the engorged ticks were also adversely impacted, which resulted in a decline in the vectors' mass [18]. Additionally, developmental abnormalities were observed. These abnormalities included the failure of nymph metamorphosis as well as tissue damage in the various tick species assessed $[17,20]$. Subolesin was discovered to be an ortholog of Akirin in insects after phylogenetic analysis showed a high degree of sequence similarity between these two proteins [21-23].

Akirin is a conserved nuclear transcription co-factor that is involved in multiple biological processes, including innate immunity [21]. Akirin regulates NF-kBdependent gene expression in the IMD pathway, as it is required at the level of the transcription factor Relish [21, 24-26]. After ingesting a blood meal, the mosquito's midgut bacterial levels increase rapidly [27]. An increase in the midgut bacterial levels causes an increase in bacterial elicitors, such as peptidoglycan (PGN) [28]. The IMD pathway is activated when PGN binds to a transmembrane peptidoglycan recognition protein known as PGRP-LC [29].

PGN induces a conformational change when binding to PGRP-LC, which causes DREDD to translocate from the nucleus to the plasma membrane [30]. DREDD-mediated cleavage of Relish allows Relish to translocate into the nucleus $[31,32]$. In the nucleus, Relish is regulated through interactions with Akirin and the BAP60 component of the Brahma (SWI/SNF) chromatin remodelling complex [21]. This allows for the expression of several antimicrobial peptide-coding genes, such as Attacin and Diptericin [33-35]. The antimicrobial peptides subsequently reduce the number of microbiotas in the mosquito's midgut to the basal level [33, 34].

The generation of homozygous null Akirin D. melanogaster mutants resulted in an embryonic lethal phenotype characteristic of defective muscle patterning, indicating that Akirin also plays a critical role in processes unrelated to immunity, such as embryogenesis [21]. When RNAi-mediated knockdown of akirin was performed, a reduction in vector survival and reproductive capacities occurred in several Culicidae species [14]. This further displayed the pleiotropic nature of Akirin and made it a potential antigen for vaccine development against various mosquito populations.

Preliminary immunisation studies were evaluated on Aedes caspius, An. atroparvus, and Culex pipiens mosquitoes [14], using immune serum that was acquired from New Zealand white rabbits that were vaccinated with recombinant Akirin from Ae. albopictus (Akirin $^{\text {albopictus }}$ ). Ingestion of the hyperimmune serum resulted in a decrease of vector survivorship [14], which provided the impetus needed for subsequent recombinant Akirin ${ }^{\text {albopictus }}$ immunisation studies. Mice were thereafter vaccinated with recombinant Akirinalbopictus to evaluate the effect of antigen-specific antibodies on Ae. albopictus and An. coluzzii life parameters [36, 37]. The ingestion of Akirin ${ }^{\text {albopictus }}$ antibodies diminished reproductive capacities of the vectors; however, it failed to reduced vector survivorship 8 days post-feeding $[36,37]$. Another antigen, Q38, was also evaluated on Ae. albopictus. The Q38 antigen was a chimera that was designed by combining protective epitopes from I. scapularis Subolesin and Ae. albopictus Akirin [38]. This antigen was used to evaluate the possibility of using Q38 as a multi-target arthropod vaccine [38]. The ingestion of Q38 antigen-specific antibodies consequently reduced Ae. albopictus' reproductive capacities as well as survivorship.

The effects of Akirin immunisation have yet to be evaluated in An. arabiensis, which is the main malaria vector in South Africa [39] and other sub-Saharan countries [1]. Anopheles arabiensis shows discrepancies in feeding behaviour (endophagic, exophagic) and resting behaviour (endophilic, exophilic) when compared to other Anopheles malaria vector species [2]. This behavioural plasticity allows An. arabiensis to evade current control methods. Therefore, alternative and additional tools, such as zooprophylaxis, are needed to supplement the current vector control methods to aid in reducing malaria transmission.

RNA-interference mediated knockdown of akirin in An. arabiensis reduced vector survivorship and reproductive capacities [40], supporting the use of Akirin as 
a potential antigen for vaccine development against this malaria vector. To determine whether Akirin could represent a novel target for the control of An. arabiensis, the efficacy of Akirin ${ }^{\text {albopictus }}$ [36] and recombinant Q38 [38] was evaluated on An. arabiensis life parameters. However, Aedes are vectors of diseases such as dengue fever, yellow fever, chikungunya, and Zika virus, among many others, which are unrelated to malaria. Therefore, an additional species-specific recombinant antigen was also evaluated on An. arabiensis, namely recombinant Akirin from An. arabiensis (Akirin ${ }^{\text {arabiensis }}$ ). This was the first time to our knowledge that a species-specific recombinant protein has been evaluated for the genus Anopheles.

All three recombinants were evaluated on $\mathrm{An}$. arabiensis concurrently to evaluate whether a species-specific vaccine will be advantageous. When evaluated, all three antigens successfully reduced An. arabiensis survival and reproductive capacities, with a vaccine efficacy of $68-73 \%$. This highlighted that the immunisation of hosts, using these protective antigens, could be further assessed to aid as a supplementary tool for An. arabiensis vector control.

\section{Methods}

\section{Biological material}

A laboratory strain of An. arabiensis mosquitoes (MBN) was used. This strain was originally colonised from wild material collected in Mamfene, northern KwaZulu-Natal, South Africa, in 2002 [40, 41].

The colony is maintained under conditions of $80 \%$ ( $\pm 5 \%$ ) humidity, $25{ }^{\circ} \mathrm{C}\left( \pm 2{ }^{\circ} \mathrm{C}\right)$, and a 12 -h day/night cycle with 45-min dusk/dawn transitions in the Botha De Meillon Insectary, at the Vector Control Reference Laboratory (VCRL) of the National Institute for Communicable Diseases (NICD) in Johannesburg [40, 42]. All adult mosquitoes were sustained on a $10 \%$ sucrose solution diet.

Two blood meals were provided to mated female mosquitoes, 5 days apart, from immunised balb/c mice. The balb/c colony was sourced from and maintained at the South African Vaccine Producers (SAVP) animal unit of the NICD, at a temperature range of $21{ }^{\circ} \mathrm{C}\left( \pm 2{ }^{\circ} \mathrm{C}\right)$, with a 12-h day/night cycle. Animal handling, injections, and veterinary procedures were strictly conducted by trained South African Veterinary Council (SAVC) registered staff. The mice did not suffer any ill effects during this study.

\section{Design and produced recombinant Akirin vaccines}

RNA was extracted from 1-day-old female An. arabiensis mosquitoes (60 mg), using Tri Reagent (SigmaAldrich, T9424), and purified using the RNeasy ${ }^{\circledR}$ MinElute $^{\mathrm{TM}}$ clean-up kit (Qiagen, 74204). A NanoDrop ${ }^{\mathrm{TM}}$ spectrophotometer was used to assess the concentration of the purified RNA, and the integrity of the RNA was assessed using a $1 \%$ non-denaturing agarose gel in TBE (Tris/Borate/EDTA), which was electrophoresed for $30 \mathrm{~min}$ at $70 \mathrm{~V}$.

The access RT-PCR system (Promega, A1250) was used to reverse-transcribe RNA into cDNA and amplify the coding region of Akirin (GenBank: KU973613). The forward primer (5'CACCATGGCGTGCGCAACGTTA3') was used to facilitate directional cloning in the pET $\mathrm{TOPO}^{\circledR}$ vector (Invitrogen, K101-01), while the reverse primer (5'GGAGAGGTAACTTGGCGCTGCTTC3') allowed the PCR product to be cloned in frame with the V5 epitope and the $6 \times$ His-tag in the pET101/D-TOPO ${ }^{\circledR}$ vector (Invitrogen, K101-01). The RT-PCR product was purified using the MinElute ${ }^{\circledR}$ PCR purification kit (Qiagen, 28004), quantified using the NanoDrop ${ }^{\mathrm{TM}}$ spectrophotometer, assessed using a $1 \%$ agarose gel in TAE (Tris/Acetate/EDTA), and sent for sequencing (Secugen SL, Madrid, Spain).

The purified RT-PCR product was cloned into the pET101/D-TOPO $^{\circledR}$ vector (Invitrogen, K101-01), after which the $\mathrm{pET}$ TOPO ${ }^{\circledR}$ construct was transformed into One Shot ${ }^{\circledR}$ TOP10 chemically competent Escherichia coli cells (Invitrogen, C6010). The transformed cells were spread on Lysogeny broth (LB) agar plates, containing $50 \mu \mathrm{g} / \mathrm{ml}$ ampicillin. After overnight incubation $\left(37^{\circ} \mathrm{C}\right)$, the GenJet ${ }^{\mathrm{TM}}$ plasmid miniprep kit (Invitrogen, K0502) was used to isolate and purify the plasmid DNA from selected colonies. Purified plasmids were quantified using the NanoDrop ${ }^{\mathrm{TM}}$ spectrophotometer and sent for sequencing.

The selected, purified plasmid was transformed into recombinant E. coli BL21 Star ${ }^{\mathrm{TM}}$ (DE3) One Shot ${ }^{\circledR}$ cells (Invitrogen, C6010-03). The transformation reaction was inoculated in LB media, containing $50 \mu \mathrm{g} / \mathrm{ml}$ ampicillin and $0.5 \%$ glucose (CONDA, 21528). After an overnight incubation $\left(37{ }^{\circ} \mathrm{C}, 200 \mathrm{rpm}\right)$, the E. coli cells, containing the plasmid with the recombinant Akirin $^{\text {arabiensis }}$, were added to a $50 \%$ glycerol solution (1:1) and stored at $-80{ }^{\circ} \mathrm{C}$, at the Instituto de Investigación en Recursos Cinegéticos, IREC, Ciudad Real, Spain, for future use.

Glycerol stocks containing recombinant Akirin $^{\text {arabiensis }}$, recombinant Akirin ${ }^{\text {albopictus }}$ (GenBank: KU973617.1) [14], and recombinant Q38 (GeneBank: JX193856.1) [38] plasmids were expressed using the $E$. coli expression system [43]. The glycerol stocks were inoculated into LB media containing $50 \mu \mathrm{g} / \mathrm{ml}$ ampicillin and $0.5 \%$ glucose and were propagated overnight $\left(37^{\circ} \mathrm{C}, 200 \mathrm{rpm}\right)$ to a final $\mathrm{OD}_{600 \mathrm{~nm}}$ of 0.4 . Isopropyl$\beta$-D-1-thiogalactopyranoside (IPTG) (Sigma-Aldrich, 367-93-1) was added to the propagated culture to a final concentration of $0.5 \mathrm{mM}$. The culture was incubated for 
an additional $5 \mathrm{~h}\left(37^{\circ} \mathrm{C}, 200 \mathrm{rpm}\right)$ to induce heterologous protein expression $\left(\mathrm{OD}_{600 \mathrm{~nm}}=1\right)$. Centrifugation was conducted to harvest the bacterial cells $(3900 \times g$, 15 min, $4{ }^{\circ} \mathrm{C}$ ).

Harvested cells were resuspended in lysis buffer (50 mM potassium phosphate, $\mathrm{pH} 7.8,400 \mathrm{mM} \mathrm{NaCl}$, $100 \mathrm{mM} \mathrm{KCl}, 7 \mathrm{M}$ urea, $10 \mathrm{mM}$ imidazole), which contained a protease inhibitor (Roche, 04693132001). The resuspended cells were mechanically disrupted by sonication (30\% amplitude, 30-s on/off pulses) using the Ultrasonic Homogenizer (Bandelin Sonopuls, Model MS73). After sonification, centrifugation was conducted $\left(15,000 \times g, 15 \mathrm{~min}, 4^{\circ} \mathrm{C}\right)$. The supernatant containing the soluble recombinant protein was purified by nickel affinity chromatography. A $1-\mathrm{ml}$ HisTrap $^{\text {TM }}$ FF column (GE Healthcare, 11-0012-38 AH) was mounted on the ÄKTAFPLC system (GE Healthcare, ÄKTA prime plus), where fractions were eluted in elution buffer $\left(50 \mathrm{mM} \mathrm{KH_{2 }} \mathrm{PO}_{4}\right.$ $\mathrm{pH} 7.8,400 \mathrm{mM} \mathrm{NaCl}, 100 \mathrm{mM} \mathrm{KCl}, 7 \mathrm{M}$ urea, $500 \mathrm{mM}$ imidazole). Eluted fractions, containing the purified protein, were transferred into a cellulose dialysis tubing membrane (Sigma-Aldrich, 3110), and incubated in $1 \times$ PBS $\left(137 \mathrm{mM} \mathrm{NaCl}, 2.7 \mathrm{mM} \mathrm{KCl}, 10 \mathrm{mM} \mathrm{Na}_{2} \mathrm{HPO}_{4}\right.$, $\left.1.8 \mathrm{mM} \mathrm{KH}_{2} \mathrm{PO}_{4} \mathrm{pH} 7.4\right)$ for $12 \mathrm{~h}$ at $4{ }^{\circ} \mathrm{C}$.

The purified recombinant proteins were quantified using the Pierce ${ }^{\mathrm{TM}}$ BCA protein assay kit (ThermoFisher Scientific, 23225), where the total protein quantification was determined using a plate reader $(562 \mathrm{~nm})$. A sodium dodecyl sulphate polyacrylamide gel electrophoresis (SDS-PAGE) was used to assess the integrity of the proteins. For each recombinant protein, $10 \mu \mathrm{g}$ of total protein was loaded on the $12 \%$ SDS-PAGE precast gel (C.B.S. Scientific, BK01212-10) with the Spectra ${ }^{\mathrm{TM}}$ multicolour broad range protein ladder (ThermoFisher Scientific, 26634) and was electrophoresed ( $1 \mathrm{~h}, 180 \mathrm{~mA})$. The gel was either stained with Blue BANDit ${ }^{\text {TM }}$ (VWR Life Science, K217) for $1 \mathrm{~h}$ or was used for Western blot analysis. After staining, the SDS-PAGE gel was washed overnight in distilled water and visualized using the gel doc system.

For Western blot analysis, the SDS-PAGE gel was transferred to a nitrocellulose membrane. The membrane was blocked with 5\% BSA for $2 \mathrm{~h}$ at room temperature, washed four times with Tris-buffered saline (TBS; $50 \mathrm{mM}$ Tris-Cl, pH 7.5, $150 \mathrm{mM} \mathrm{NaCl}, 0.5 \%$ Tween 20), and incubated with anti-His ${ }_{6}$ from mouse IgG $_{1}$ (SigmaAldrich, 11922416001) at a 1:500 dilution in TBS. The membrane was incubated overnight at $4{ }^{\circ} \mathrm{C}$. Thereafter, the membrane was washed four times with TBS and incubated with an anti-mouse IgG-horseradish peroxidase (HRP) conjugate (Sigma-Aldrich, AP308P) that was diluted to 1:1000 in TBS with 3\% BSA. The membrane was washed five times with TBS and finally developed with tetramethylbenzidine (TMB) stabilised substrate for
HRP (Promega, W4121), according to the manufacturer's recommendations.

\section{Mouse immunisation}

The recombinant proteins were concentrated to a final concentration of $250 \mu \mathrm{g} / \mathrm{ml}$ in PBS and emulsified with $1.5 \mathrm{ml}$ of adjuvant (Montanide ${ }^{\mathrm{TM}}$, ISA $50 \mathrm{~V} 2$ ) to formulate the recombinant vaccines. A vaccine consisting of PBS and adjuvant was used as a negative control. Five-weekold balb/c mice were vaccinated intraperitoneally, using a double-blind approach, where each treatment was given a code and the identity of the code was not revealed to the researchers until the end of the experiment. Therefore, mice were either vaccinated with recombinant protein (25 $\mu \mathrm{g} / \mathrm{dose})$ or the control consisting of PBS and adjuvant ( 1 mouse per treatment, 4 treatments, 3 replicates, $n=12$ mice). Each mouse received three doses of vaccine, which were administered 2 weeks apart.

Two weeks after the final vaccination, the immunised mice were used to provide female An. arabiensis mosquitoes with two subsequent blood meals. This was done because the mosquito colony was routinely provided with two blood meals before ovipositioning. Therefore, the female mosquitoes were 5 days old at the first feeding and 9 days old at the second feeding. Prior to blood-feeding, female mosquitoes were mated with male mosquitoes over 4 days, using a male to female ratio of $1: 1$, after which the male mosquitoes were removed from the cages.

Histamine present in the mosquito's salivary glands did not induce an itch-associated response in mice; thus, the mice did not experience discomfort after blood-feeding [44]. However, to prevent discomfort during feeding, the mice were anaesthetised with a mix of Xylazine and Anaket $(0.02-0.03 \mathrm{ml})$. Once anaesthetised, each mouse was placed on top of their respective mosquito cage (BugDorm, 4M1515). Each cage retained a total of 100 mated female mosquitoes, which were starved $12 \mathrm{~h}$ before feeding. The mosquitoes fed for a total of $35 \mathrm{~min}$, after which the mice were removed from the top of each cage. The mosquitoes had a feeding success of approximately $80 \%$ per feeding, which was a $66.3 \%$ overall feeding success per treatment ( 2 feedings, 3 replicates, $n=199$ mosquitoes). Mosquitoes that did not take a blood meal were subsequently removed from the study after feeding; thus, both fully and partially fed mosquitoes were included in the analysis of this study. This would simulate similar results to those of a field setting.

\section{Mosquito life table parameters}

The rate of survival was monitored until $100 \%$ mortality was reached for all four treatments, after which a KaplanMeier survival curve was constructed using Statistix 10. 
A log-rank test was performed to determine whether there was a statistically significant difference in rate of survival between the treatments $(p<0.05)$ [40]. Simultaneously, vector fecundity and vector fertility were also analysed. Two days after receiving the second blood meal, filter paper egg plates were placed at the bottom of each cage. The eggs laid on each plate were counted daily, over 5 days. Once the eggs were counted, they were placed into separate plastic containers, filled with $500 \mathrm{ml}$ of $\mathrm{dH}_{2} \mathrm{O}(\mathrm{L}: 226 \mathrm{~mm}, \times \mathrm{W}: 166 \mathrm{~mm} \times \mathrm{H}: 10 \mathrm{~mm})$. The hatchlings were counted over 14 days. Vector fecundity was defined by determining the mean number of eggs laid per female mosquito, and vector fertility was defined by determining the mean percentage of hatchlings. A one-way ANOVA test and a Tukey-HSD all pairwise comparison test were used to compare the mean fecundity and fertility between the treatments $(p<0.05)$ [40].

Vaccine efficiency was used to interpret the overall effect of each antigen on the An. arabiensis population tested. Vaccine efficiency is a good method to compare the effect of different antigens on a vector population; however, this parameter cannot be compared between vector species, as their developmental processes may differ [5]. Efficiency (E) was calculated considering the effect of vaccination on the reduction of the mean vector survival rate $(\mathrm{S})$, vector fecundity $(\mathrm{Fc})$, and vector fertility $(\mathrm{Fr})$ as $\mathrm{E}(\%)=100[(1-\mathrm{S} / 100)(1-\mathrm{Fc} / 100)(1-\mathrm{Fr} / 100)]$ [16]. A one-way ANOVA test and Tukey-HSD all pairwise comparison test were used to compare the vaccine efficacy between the treatments $(p<0.05)$.

\section{Sample collection}

Blood samples were collected from the saphenous vein [45] of each mouse $24 \mathrm{~h}$ before each immunisation and blood-feeding. The vein was punctured using a 27.5gauge needle, where drops of blood were collected. Centrifugation was conducted on the blood samples to separate the serum from the red blood cells $(1500 \times g$, $10 \mathrm{~min}, 4{ }^{\circ} \mathrm{C}$ ). Thereafter, the serum was used to perform an indirect ELISA to determine the antibody titres within the mice. Simultaneously, three engorged mosquitoes were randomly selected from each treatment 24 $\mathrm{h}$ after the final blood-feeding ( 3 mosquitoes per replicate, 3 replicates, $n=9$ mosquitoes per treatment). These mosquitoes were used to determine whether antibodies from the vaccinated mice were ingested by the engorged females and whether these antibodies could subsequently be detected when performing an indirect ELISA.

\section{Antibody detection}

Each recombinant protein was diluted in $100 \mu \mathrm{l}$ Tris-buffered saline ( $25 \mathrm{mM}$ Tris $\mathrm{HCl}, 150 \mathrm{mM} \mathrm{NaCl}, 2 \mathrm{mM} \mathrm{KCl}$ ) (TBS) and used to coat a separate 96-well microplate
$(0.1 \mu \mathrm{g})$. The recombinant proteins were immobilised to the microplate by overnight incubation $\left(4{ }^{\circ} \mathrm{C}\right)$. Thereafter, the microplates were blocked with Blocker ${ }^{\mathrm{TM}}$ BLOTTO (ThermoFisher Scientific, 37530) for $2 \mathrm{~h}$ at $37{ }^{\circ} \mathrm{C}$. The blocking solution was aspirated off the microplate. The immobilised recombinant proteins were paired to the respective immunogen for the determination of the respective antibody titres, where each treatment was assessed in duplicate. Samples consisting of TBS were used as background controls.

The mouse sera samples or the TBS control samples were diluted in blocking solution $(1: 20, \mathrm{v} / \mathrm{v})$ and hybridised to the microplate $\left(2 \mathrm{~h}, 37^{\circ} \mathrm{C}\right)$ to quantify the antibody titres within the mice. For the quantification of antibodies ingested by the mosquitoes, the blood-fed mosquitoes were homogenized in $200 \mu \mathrm{l}$ TBS-Tween 20 (TBS-T) and centrifuged to collect the supernatant (3 $\mathrm{min}, 16,000 \times g, 4{ }^{\circ} \mathrm{C}$ ). The supernatant was subsequently hybridised to the microplate $\left(2 \mathrm{~h}, 37^{\circ} \mathrm{C}\right)$. After hybridisation, the microplate was washed three times with TBS-T. Goat anti-mouse IgG antibody, conjugated with horseradish peroxidase (HRP) (Agilent, P0447), was diluted in blocking buffer (1:3000). The diluted secondary antibody was bound to the primary antibody through a 2-h incubation $\left(37^{\circ} \mathrm{C}\right)$. Unbound secondary antibody was aspirated off the microplate. The microplate was then washed three times with TBS-T before adding the SigmaFast $^{\mathrm{TM}}$ enzyme-substrate (Sigma-Aldrich, N1891).

After a 30-min incubation period, the microplate was analysed at $450 \mathrm{~nm}$ using the multi-well plate reader. The antibody titres were represented as OD ${ }_{450 \mathrm{~nm}}$ (OD mouse serum or mosquito sample - OD TBS control). The antibody titres were compared between the control and treated groups, using an unpaired $t$ test $(p<0.05)$. Additionally, the fold change of the antibody titres was calculated between the treatments and control group using the formula "(B-A)/A". A Pearson correlation analysis was also conducted in Microsoft Excel to compare the significant effects of antibody response on An. arabiensis lifetable parameters $(r<-0.5)$.

\section{Results}

\section{Mouse antibody detection}

The recombinant proteins were successfully produced using the E. coli expression system (Additional file 1), emulsified with the adjuvant to formulate the recombinant vaccines, and administered to the balb/c mice. The antibody titres of the mice that were vaccinated with the recombinant proteins were significantly higher than those of the control mice. The mean antibody titres of the mice that were vaccinated with the recombinant Q38 protein were 0.7 -fold higher than the mean antibody titres of the control mice (unpaired $t$ test: $t=2.46$, 
$p=0.05, \mathrm{DF}=1)($ Fig. 1a). Similarly, the antibody titres of the mice vaccinated with the recombinant Akirin ${ }^{\text {arabiensis }}$ protein were 0.9 -fold higher than those of the control mice (unpaired $t$ test: $t=2.98, p=0.03, \mathrm{DF}=1$ ) (Fig. 1b), and the antibody titres of the mice vaccinated with the recombinant Akirin ${ }^{\text {albopictus }}$ protein were 0.5 -fold higher than those of the control mice overall (unpaired $t$ test: $t=2.90, p=0.03, \mathrm{DF}=1$ ) (Fig. 1c).

\section{Mosquito antibody detection}

The female An. arabiensis mosquitoes successfully ingested the antibodies from the vaccinated mice. The antibody titres of the females that ingested blood from the mice vaccinated with recombinant protein were significantly higher than the antibody titres of females that ingested blood from the control mice ( 3 mosquitoes per replicate, 3 replicates, $n=9$ mosquitoes per treatment). When the antibodies titres were quantified, it was seen that the An. arabiensis females who ingested the antiQ38 antibodies had an antibody titre that was 1.5-fold higher than the females who ingested blood from the control mice (unpaired $t$ test: $t=3.25, p=0.03, \mathrm{DF}=1$ ). The An. arabiensis females who ingested the anti-Akirin $^{\text {arabiensis }}$ antibodies had an antibody titre that was 2.6fold higher than the females who ingested blood from the control mice (unpaired $t$ test: $t=7.04, p<0.002, \mathrm{DF}=1$ ), and the An. arabiensis females who ingested the antiAkirin ${ }^{\text {albopictus }}$ antibodies had an antibody titre that was 8.5-fold higher than the females who ingested blood from the control mice (unpaired $t$ test: $t=11.92, p<0.001$, $\mathrm{DF}=1)$.

\section{Mosquito lifetable parameters}

Vector survival was significantly reduced in An. arabiensis females that ingested blood from the mice that were vaccinated with recombinant proteins compared to the females that ingested blood from the control mice (logrank: $\mathrm{X}^{2}=84, p<0.01, \mathrm{DF}=3$ ) (Additional file 2). Anopheles arabiensis that ingested blood from the control mice had a mean survival time of 21 days, while the females

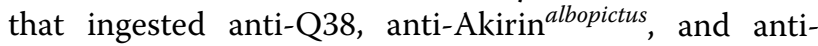
Akirin $^{\text {arabiensis }}$ antibodies had a mean survival time of 16 , 14 , and 13 days respectively. This was a $24 \%, 33 \%$, and $38 \%$ reduction in mean survival time compared to the control treatment (Fig. 2).

Vector fecundity was also significantly reduced in the An. arabiensis females that ingested blood from the mice vaccinated with recombinant proteins compared to the females that ingested blood from the control mice (oneway ANOVA: $p<0.001, F=11.7, \mathrm{DF}=3)$. Anopheles arabiensis that ingested blood from the control mice had a mean fecundity of 66 eggs laid per female mosquito, while the females that ingested anti-Q38, anti-Akirin ${ }^{\text {albop- }}$ ictus, and anti-Akirin ${ }^{\text {arabiensis }}$ antibodies had a mean fecundity of 31,38 , and 33 eggs laid per female, respectively. This was a $53 \%, 42 \%$, and $50 \%$ reduction in mean fecundity compared to the control treatment (Fig. 3).

Similarly, vector fertility was also significantly reduced in the An. arabiensis females that ingested blood from the mice vaccinated with the recombinant proteins compared to the females that ingested blood from the control mice (one-way ANOVA: $p<0.001, \mathrm{~F}=68.9, \mathrm{DF}=3$ ). Anopheles arabiensis that ingested blood from the control mice had a $91 \%$ overall hatch rate of the eggs that were laid per female mosquito, while the females that ingested anti-Q38, anti-Akirin ${ }^{\text {albopictus }}$, and anti-Akirin $^{\text {arabiensis }}$ antibodies had an overall hatch rate of $82 \%$, $73 \%$, and $70 \%$, respectively. This was a $10 \%, 23 \%$, and $20 \%$ reduction in mean fertility compared to the control treatment (Fig. 3).

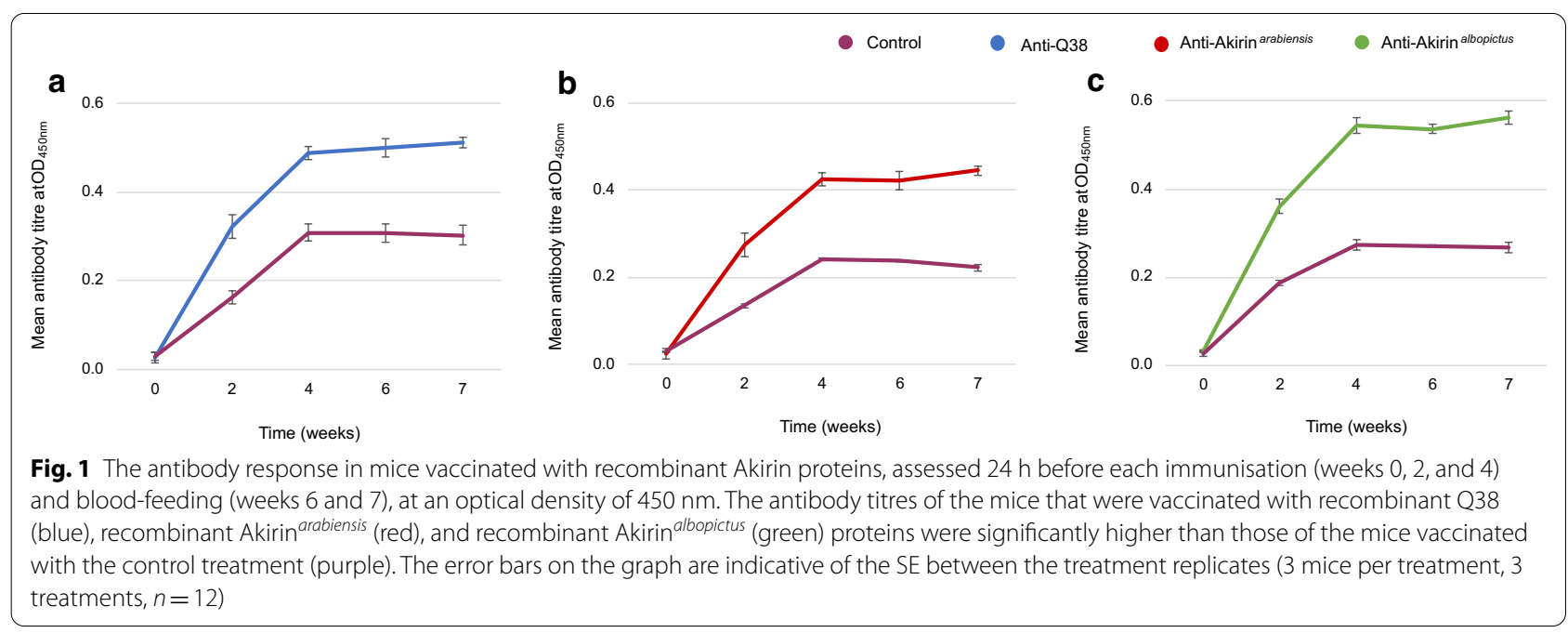




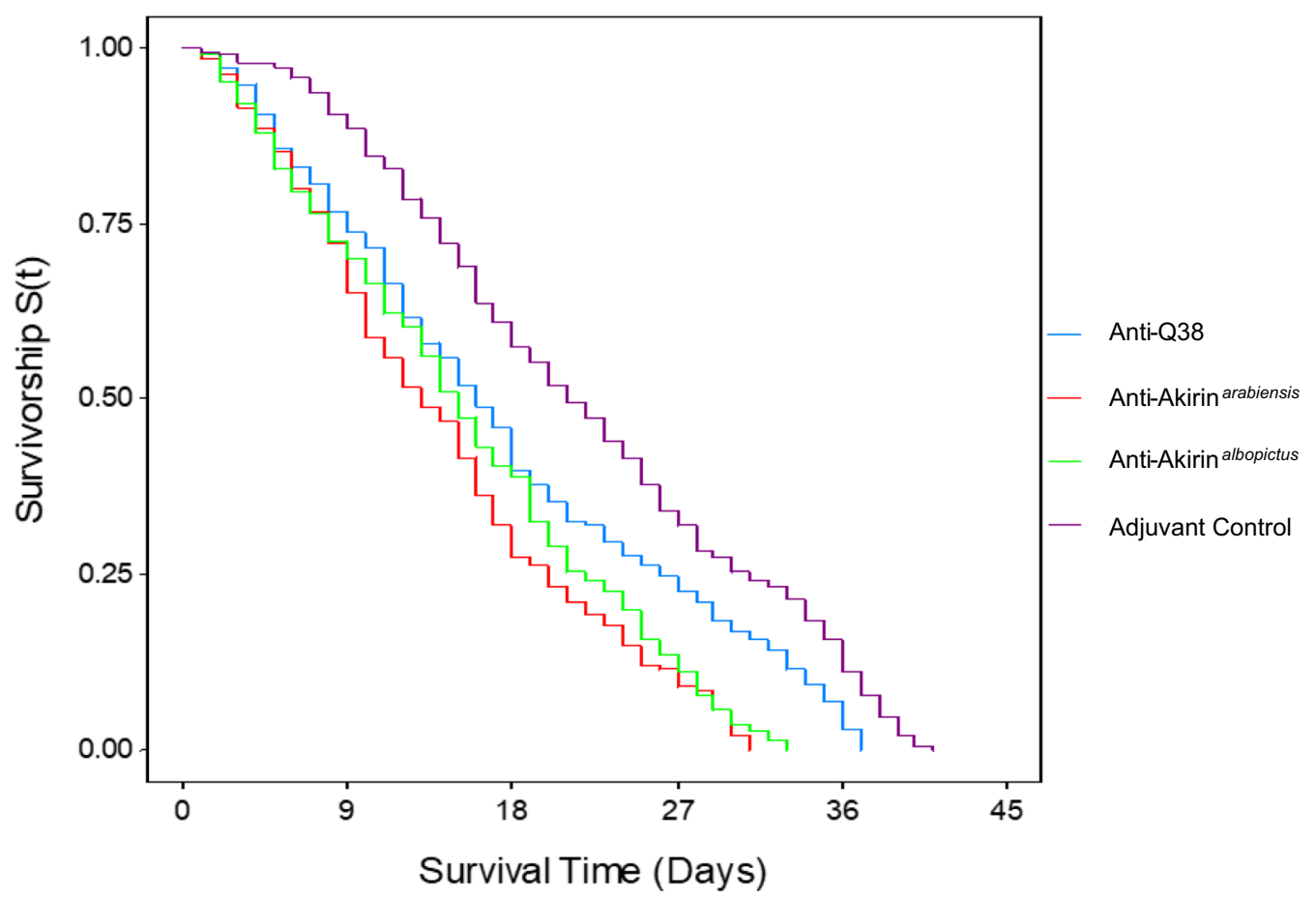

Fig. 2 Kaplan-Meier survival analysis of the female An. arabiensis mosquitoes assessed post-blood-feeding (190 mosquitoes per treatment). The females that ingested anti-Q38 antibodies (blue), anti-Akirin albopictus antibodies (green), and anti- Akirin arabiensis antibodies (red) reached 100\% mortality 4,8 , and 10 days (respectively) before the females that ingested blood from the control mice (purple). Vector survival was significantly reduced in the females that ingested blood from the mice vaccinated with recombinant proteins compared to the females that ingested blood from the control mice (log-rank: $x^{2}=84, p<0.01, \mathrm{DF}=3$ )
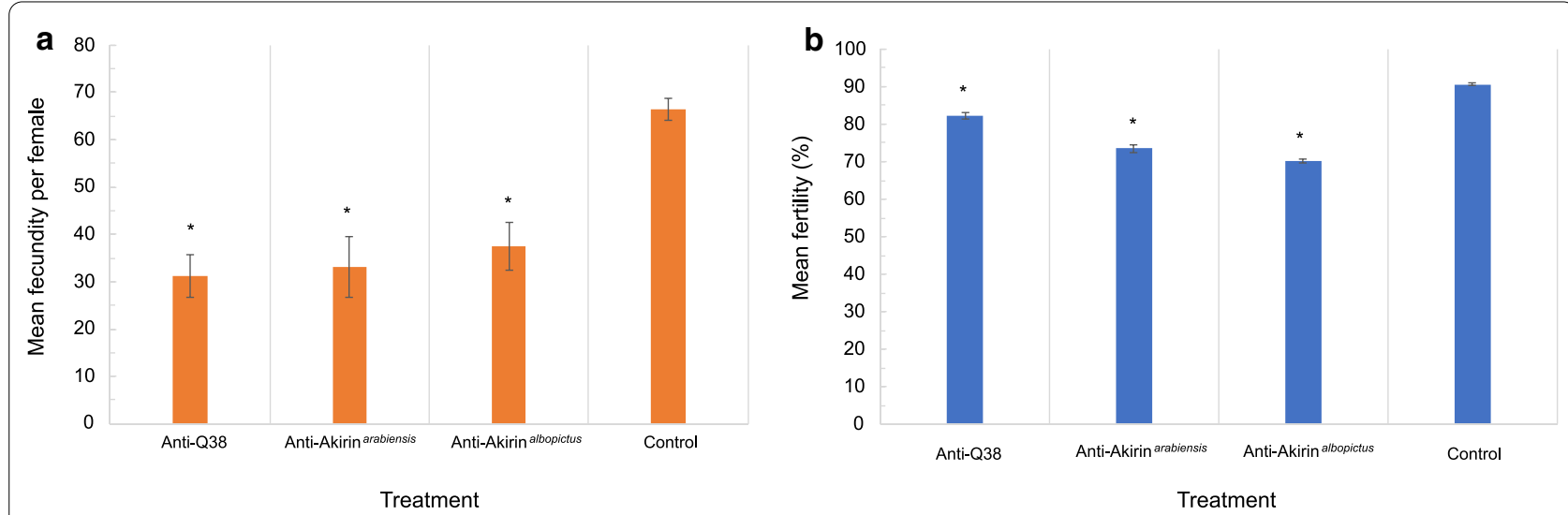

Fig. 3 The mean vector fecundity (a) and vector fertility (b) assessed per female An. arabiensis mosquito ( $n=190$ per treatment), post-blood-feeding. Vector fecundity (one-way ANOVA: $p<0.001, F=11.7, \mathrm{DF}=3$ ) and vector fertility (one-way ANOVA: $p<0.001, \mathrm{~F}=68.9, \mathrm{DF}=3$ ) were significantly reduced in An. arabiensis females that ingested antibodies from the mice that were vaccinated with the recombinant proteins compared to the control treatment. The asterisk shows a significant change from the control treatment, while the error bars on the graph are indicative of the SE between the treatment replicates (Additional file 3)

When comparing the lifetable parameters between the various treatments, it was seen that the An. arabiensis populations that ingested the antibodies from the mice vaccinated with recombinant proteins were adversely affected compared to the An. arabiensis mosquitoes that ingested blood from the control mice (Table 1). Overall, the recombinant Q38 antigen had a vaccine efficacy of $67.8 \%$ on the targeted An. arabiensis vector population, 
Table 1 A comparison of the lifetable parameters between the An. arabiensis female mosquitoes that ingested blood from the control mice and the An. arabiensis females that ingested blood from the mice that were vaccinated with recombinant Q38, Akirin arabiensis, and Akirin ${ }^{\text {albopictus }}$ proteins

\begin{tabular}{|c|c|c|c|c|}
\hline Treatment & Control & Q38 & Akirin arabiensis & Akirin albopictus \\
\hline \multirow[t]{4}{*}{ Mean survival rate (days) $\pm S D ; N$} & 22 & 14 & 12 & 15 \\
\hline & 21 & 15 & 12 & 14 \\
\hline & 20 & 18 & 15 & 15 \\
\hline & $(21 \pm 1 ; 190)^{\mathrm{a}}$ & $(16 \pm 2 ; 190)^{b}$ & $(13 \pm 2 ; 190)^{b}$ & $(14 \pm 1 ; 190)^{b}$ \\
\hline \multirow{4}{*}{$\begin{array}{l}\text { Fecundity (eggs laid/ total amount of } \\
\text { females) } \pm \text { SD; N }\end{array}$} & 68 & 37 & 29 & 28 \\
\hline & 70 & 22 & 25 & 40 \\
\hline & 62 & 34 & 46 & 45 \\
\hline & $(66 \pm 4 ; 190)^{a}$ & $(31 \pm 8 ; 190)^{b}$ & $(33 \pm 11 ; 190)^{b}$ & $38 \pm 9 ; 190)^{b}$ \\
\hline \multirow[t]{4}{*}{ Fertility (eggs hatched/eggs laid) $\pm S D ; N$} & 0.9 & 0.8 & 0.7 & 0.7 \\
\hline & 0.9 & 0.8 & 0.7 & 0.7 \\
\hline & 0.9 & 0.8 & 0.8 & 0.7 \\
\hline & $(0.9 \pm 0.1 ; 190)^{\mathrm{a}}$ & $(0.8 \pm 0.1 ; 190)^{b}$ & $(0.7 \pm 0.2 ; 190)^{c}$ & $0.7 \pm 0.1 ; 190)^{c}$ \\
\hline Vaccine efficacy (\%) & - & $67.8^{\mathrm{a}}$ & $73.2^{\mathrm{a}}$ & $72.4^{\mathrm{a}}$ \\
\hline
\end{tabular}

*The results are shown for each vaccine trial ( $n=3$ replicates), with the average standard deviation $( \pm$ SD) and the total sample size $(\mathrm{N})$ assessed. The data were analysed using either the log-rank test $(p<0.05)$ or a one-way ANOVA test $(p<0.05)$. The letters "a-c" were used to represent statistical differences between the various groups when conducting the Tukey-HSD test

while the recombinant Akirin ${ }^{\text {albopictus }}$ antigen had a vaccine efficacy of $72.4 \%$, and the recombinant Akirin ${ }^{\text {arabiensis }}$ antigen had a vaccine efficacy of 73.2\% (Additional file 4).

The adverse effects observed on the An. arabiensis populations that ingested the anti-Q38, anti-Akirin ${ }^{\text {arabiensis, }}$, and anti-Akirin ${ }^{\text {albopictus }}$ antibodies negatively correlated with the mean antibody titres of the vaccinated mice (Fig. 4).

\section{Discussion}

The significant reduction in vector survival and reproductive capacities characterised by the knockdown of akirin in An. arabiensis [40] made Akirin a potential antigen for vaccine development against the untargeted outdoor vector populations. Thus, to determine whether Akirin could represent a novel target for the control of An. arabiensis, the efficacy of three recombinant vaccines was evaluated concurrently, namely recombinant Akirin $^{\text {arabiensis }}$, recombinant Akirin ${ }^{\text {albopictus }}$, and recombinant Q38. Antibodies against the recombinant proteins were effectively elicited in vaccinated mice and were successfully ingested by the engorged An. arabiensis. Thereafter, vector lifetable parameters such as fertility, fecundity, and longevity were assessed.

Anopheles arabiensis longevity was significantly reduced when anti-Akirin ${ }^{\text {arabiensis }}$ antibodies, antiAkirin $^{\text {albopictus }}$ antibodies, and anti-Q38 antibodies were ingested. Although not completely understood, it is thought that the ingested antibodies cross the cell membrane and interact with the target protein in the cytoplasm, thus preventing Akirin from translocation to the nucleus $[8,46]$. Previous Akirin gene regulation analysis, performed in An. coluzzii, showed that akirin expression was downregulated by $16-40 \%$ in the mosquito midgut and $25-65 \%$ in the remaining tissue [37]. This shows that akirin is expressed constitutively through the mosquito's body and thus explains the profound effects observed on the vector's biology when Akirin is targeted.

As aforementioned, Akirin regulates NF-kB dependent gene expression in the IMD pathway, which allows for the gene expression of several antimicrobial peptidecoding genes [21, 25, 26, 33-35]. Since the antimicrobial peptides reduce the number of microbiotas in the midgut to the basal level [33,34], it is possible that targeting Akirin disrupts bacterial homeostasis in An. arabiensis. Disrupting the bacterial homeostasis would weaken the innate immune defence in An. arabiensis, which would ultimately affect the survival of the vector [21, 27, 47]. However, additional research is needed to verify this hypothesis.

Mosquito longevity is a critical factor regarding malaria transmission [48]. To transmit malaria, a female Anopheles mosquito must ingest a blood meal infected with gametocytes. The gametocytes must encounter the midgut epithelium, undergo fertilization, develop into motile ookinetes, traverse across the midgut epithelium, develop into oocysts, produce haploid sporozoites, and migrate through the mosquito haemolymph to the salivary glands to complete the sporogonic cycle $[49,50]$. It takes approximately 14 days to complete this cycle. Once the sporogonic cycle is complete, the sporozoites are injected into the next vertebrate host when taking a successive blood 


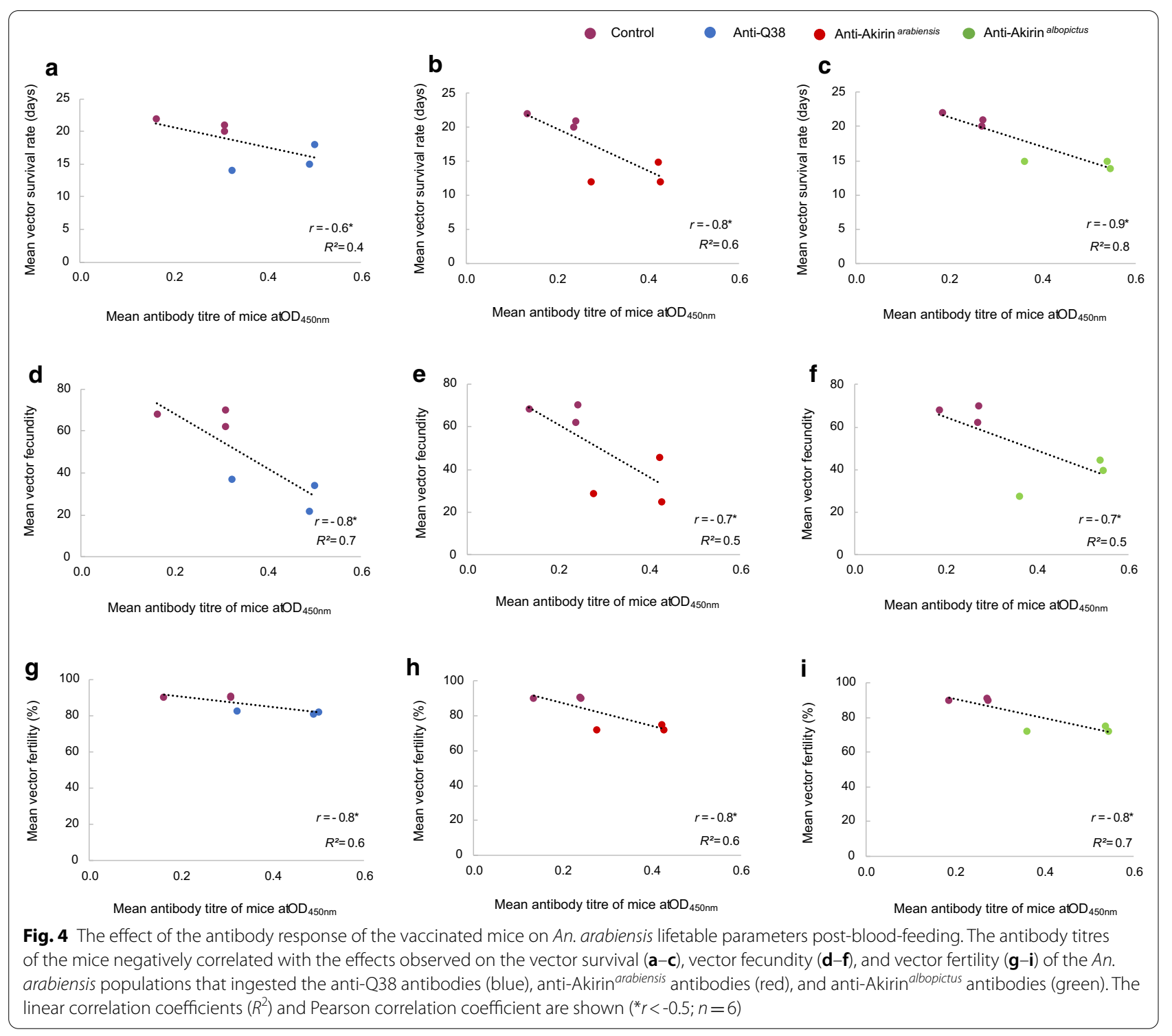

meal $[51,52]$. This allows for the transmission of malaria. When the female An. arabiensis mosquitoes ingested the anti-Q38 antibodies, anti-Akirin ${ }^{\text {albopictus }}$ antibodies, and anti- Akirin ${ }^{\text {arabiensis }}$ antibodies, they had a mean survival time of 16, 14, and 13 days, respectively. Eliminating a third of the vector population before the completion of the sporogonic cycle could significantly affect the transmission of malaria.

Additionally, both vector fecundity and vector fertility were significantly reduced when anti-Akirin ${ }^{\text {arabiensis }}$ antibodies, anti-Akirin ${ }^{\text {albopictus }}$ antibodies, and anti-Q38 antibodies were ingested by $A n$. arabiensis. It has been postulated that the movement of antibodies to reproductive tissues interferes with oogenesis, as the synthesis of vitellogenin by the fat bodies as well as its uptake of vitellogenin from the haemolymph may be inhibited [53]. Alternatively, it has been suggested that the ingested mosquito antibodies may irritate the gut, which in turn may reduce the uptake of a blood meal or the availability of nutrients. The content of some of the developing follicles may therefore be reabsorbed, resulting in reduced vector ovipositioning [53]. This, however, would need further investigation. The impact on larval development were not investigated in this study, but future studies should take this into consideration.

Akirin, however, is known to play a critical role in processes unrelated to immunity, such as development and reproduction [14, 21]. As aforementioned, immunisation of cattle, using the recombinant ortholog, Subolesin, had several deleterious effects on the engorged ticks. This 
included developmental abnormalities, such as the failure of nymph metamorphosis and tissue damage in the various tick species assessed, as well as diminished vector ovipositioning [17, 19, 20]. Regardless of the underlying mechanism, reducing the number of An. arabiensis eggs laid by half and the amount of viable $A n$. arabiensis progeny by a fifth could decrease the vector population. It will be vital to investigate the impact of the recombinant vaccine on vector susceptibility to the human Plasmodium parasite.

In this study, the female An. arabiensis mosquitoes were allowed to feed on each of the respective mice for a total of $35 \mathrm{~min}$. As aforementioned the original concept of concealed antigen identification and vaccination originated in ticks. Ticks are long-duration feeders, whereas mosquitoes are short-duration feeders. Mosquitoes, therefore, ingest less blood than ticks. However, mosquitoes ingest an additional blood meal every 2 to 3 days. This would ensure repeated ingestion of the antimosquito antibodies, which could disrupt gut bacterial homeostasis, consequently reducing the lifespan of the vector [27] as well as its reproductive fitness. Reducing both An. arabiensis survivorship and reproductive fitness would ultimately cause a reduction in the vector's population abundance. A reduction in vector abundance would have a significant impact on malaria transmission.

It should be noted that the adverse effects that were observed on the An. arabiensis population that ingested the anti-Akirin ${ }^{\text {albopictus }}$ antibodies was an interesting observation. Previous recombinant Akirin ${ }^{\text {albopictus }}$ immunisation experiments affected Ae. aegypti, Ae. albopictus, and An. coluzzii oviposition; however, the ingestion of anti-Akirin ${ }^{\text {albopictus }}$ antibodies did not affect vector longevity [36, 37]. These differences can be attributed to various experimental factors that need further investigation. The sample size that was used to assess the effect of antiAkirin vaccines on An. arabiensis was much larger than those described in past studies [36, 37]. Additionally, the previous studies only provided a single blood meal to the female mosquitoes [36, 37], while An. arabiensis were provided with two blood meals. The repeated ingestion of the anti-mosquito antibodies could have added to the adverse effects observed on the mosquito lifetable parameters. Furthermore, vector fertility was not measured in the previous studies, and vector survival was only monitored for a limited period (7/8 days post-blood-feeding). The impact of the physiological difference between the various genera and species, however, cannot be ruled out [36]. These include the sensitivity of the vector's midgut to proteases [54] as well as the volume of blood ingested by each vector species.

Another possible reason for the variability of these results could be the system that was used to make these recombinant vaccines. In previous immunisation studies, the Pichia pastoris expression system was used to express recombinant Akirin ${ }^{\text {albopictus }}$ and recombinant Q38 antigens [36-38], while this investigation used the $E$. coli expression system. The $E$. coli expression system has, however, been successfully used to express various recombinant proteins for the control of other arthropod studies [43]. One way to overcome this would be to standardize mosquito vaccine research, similar to that proposed for tick vaccines [55]. These parameters would need to include the standardisation of the expression system used, the type of adjuvant used, the experimental procedures followed to determine the antibody titres and vaccine efficacy, the sample size used to make these comparisons, as well the type and age of the experimental animals used within the study. This would allow for a more accurate comparison of results between different research groups.

When comparing the efficacy among the three recombinant vaccines, the difference was negligible. However, the chimeric nature of Q38 makes this antigen a valuable multi-target arthropod vaccine. Previously, the efficacy of Q38 was tested on Ixodes ricinus, Dermacentor reticulastus (ticks), and Phlebotomus perniciosus (sandflies) [38, 56]. In P. perniciosus, the ingestion of anti-Q38 antibodies significantly reduced vector oviposition by $16-26 \%$ [38]. The ingestion of anti-Q38 antibodies, by I. ricinus, significantly reduced vector longevity by 4.25 -fold and moulting by $38 \%$, while the ingestion of anti-Q38 antibodies significantly reduced the mass of $D$. reticulastus by $25 \%$ and moulting by $15 \%$ [56]. These previously reported findings, as well as those observed for the ingestion of anti-Q38 antibodies, which significantly reduced the reproductive capacities and vector survivorship of $A e$. albopictus [38] and An. arabiensis, demonstrate the ability of using this recombinant vaccine to target multiple ectoparasitic infestations simultaneously using various agricultural blood hosts.

The conserved structure and function of Akirin throughout the metazoan $[17,25]$ have given rise to the question of safety when vaccinating vertebrates because of autoimmunity. As in previous experiments with different hosts, physiological or pathological alterations were not observed in vaccinated mice [14]. This suggests that, as expected, the antibody response was directed against non-self-epitopes, thus reducing the possibility of detrimental effects to the vaccinated host. Furthermore, Akirin is an intracellular antigen and, through still unknown mechanisms, the antibody can enter arthropod but not mammalian cells [8]. Additionally, effective immunisation of various hosts, for the control of numerous vector infestations, displays the low risk of inducing an autoimmune response in vertebrates $[14,36,38,43]$. 
Challenging various blood hosts with whole protein or antigenic peptides may provide a valuable intervention against several ectoparasites including the untargeted exophagic and exophilic An. arabiensis mosquito populations. This vector control strategy could also impact secondary malaria vectors, or vectors of other diseases, if similar detrimental effects are caused within these vectors. Further studies, however, still need to be performed to determine if agricultural or domestic animals, vaccinated with recombinant Akirin vaccines, can elicit a similar response against An. arabiensis to those observed when recombinant Akirin vaccines were evaluated using balb/c mice. Furthermore, the half-life of these antibodies will need to be assessed within the reservoir hosts to determine how often booster vaccines will need to be administered to the agricultural or domestic animals.

This form of zooprophylaxis could therefore be applied in combination with other vector control strategies to divert An. arabiensis away from human hosts. Simultaneously, the $A n$. arabiensis vector population would be targeted when feeding on these hosts by ingesting the anti-Akirin antibodies. This would cause a reduction of vector survivorship and reproductive capacities. By reducing An. arabiensis survivorship as well as reproductive fitness, a reduction in vector population abundance would ultimately occur. A reduction in vector abundance would have a significant impact on malaria transmission, especially in countries with low-level seasonal malaria transmission, such as South Africa.

\section{Conclusions}

The results reported here were the first showing that hosts vaccinated with recombinant Akirin ${ }^{\text {arabiensis }}$ can develop a protective response against mosquito populations. Hosts vaccinated with recombinant Akirin albopictus and recombinant $\mathrm{Q} 38$ also developed a protective response against $A n$. arabiensis, and thus existing vaccines could be used to target this vector population. The reduction in An. arabiensis survival and reproductive capacities, due to revivor-host immunisation, has provided a step toward the development of a novel target for the control of An. arabiensis.

\section{Supplementary Information}

The online version contains supplementary material available at https://doi. org/10.1186/s13071-021-04711-8.

Additional file 1. Data for survival analysis.

Additional file 2. Fecundity and fertility dataset.

Additional file 3. Calculation for vaccine efficacy.

Additional file 4. Overall vaccine efficacy.
Acknowledgements

Not applicable.

\section{Authors' contributions}

Conceptualization, LLK; methodology, BDL; LLK; YD; MC; JdIF; software, BDL; MC; validation, LLK; YD; MC; JdIF; formal analysis, BDL; MC; LLK; investigation, $\mathrm{BDL}$; IL; resources, LLK; JdlF; data curation, BDL; writing—original draft preparation, BDL; writing — review and editing, LLK; YD; MC; JdlF; IL; visualization, BDL; YD; LLK; supervision, LLK; YD; MC; JdIF; project administration, LLK; funding acquisition, LLK; YD; JdIF. All authors read and approved the final manuscript.

\section{Funding}

This project was supported by funding from the National Research Foundation (NRF) Competitive Programme for Rated Researchers (CPRR), Incentive Funding (UID; 85538), and the DST/NRF Research Chair Initiative grant awarded to L.L.K. (UID: 64763), as well as the Medical Faculty Research

Endowment Fund awarded to B.D.L, and funding Y.D.M. from the South African Medical Research Council—Self-Initiated Research Grant. The funders had no role in the design of the study; in the collection, analyses, or interpretation of data; in the writing of the manuscript, or in the decision to publish the results.

\section{Availability of data and materials}

The datasets generated for this study are available as additional files.

\section{Declarations}

Ethics approval and consent to participate

Animal ethics was reviewed and approved by the University of the Witwatersrand (WITS) Animal Ethics Committee (AEC) (AESC: 2017-010-72B) as well as the National Health Laboratory Service (NHLS) AEC (AESC: 001-19).

\section{Consent for publication}

Not applicable.

\section{Competing interests}

The authors declare no competing interests.

\section{Author details}

${ }^{1}$ Wits Research Institute for Malaria, School of Pathology, Faculty of Health Sciences, University of the Witwatersrand, Johannesburg, South Africa. ${ }^{2}$ Centre for Emerging Zoonotic and Parasitic Diseases, National Institute for Communicable Diseases of the National Health Laboratory Service, Johannesburg, South Africa. ${ }^{3}$ SaBio. Instituto de Investigacion en Recursos Cinegeticos IREC-CSIC-UCLM-JCCM, Ronda de Toledo s/n, 13005 Ciudad Real, Spain. ${ }^{4}$ Interdisciplinary Laboratory of Clinical Analysis, Regional Campus of International Excellence Campus Mare Nostrum, Interlab-UMU, University of Murcia, Espinardo, 30100 Murcia, Spain. ${ }^{5}$ South African Vaccine Producers Animal Unit, National Institute for Communicable Diseases of the National Health Laboratory Service, Johannesburg, South Africa. ${ }^{6}$ Department of Veterinary Pathobiology, Center for Veterinary Health Sciences, Oklahoma State University, Stillwater, OK 74078, USA.

Received: 20 January 2021 Accepted: 31 March 2021

Published online: 20 April 2021

References

1. Sinka ME, Bangs MJ, Manguin S, Coetzee M, Mbogo CM, Hemingway J, Patil AP, Temperley WH, Gething PW, Kabaria CW, Okara RM. The dominant Anopheles vectors of human malaria in Africa, Europe and the Middle East: occurrence data, distribution maps and bionomic précis. Parasit Vectors. 2010;3(1):117.

2. Gillies MT, Coetzee M. A supplement to the Anophelinae of Africa South of the Sahara. Publ South Afr Inst Med Res. 1987;55:1-43.

3. de la Fuente J, Rodríquez M, Redondo M, Montero C, García-García J, Méndez L, Serrano E, Valdés M, Enriquez A, Canales M, Ramos E. Field studies and cost-effectiveness analysis of vaccination with GavacTM against the cattle tick Boophilus microplus. Vaccine. 1998;16(4):366-73. 
4. Valle MR, Mèndez L, Valdez M, Redondo M, Espinosa CM, Vargas M, Cruz RL, Barrios HP, Seoane G, Ramirez ES, Boue O. Integrated control of Boophilus microplus ticks in Cuba based on vaccination with the anti-tick vaccine Gavac ${ }^{\text {TM. }}$. Exp Appl Acarol. 2004;34(3-4):375-82.

5. Manzano-Román R, Díaz-Martín V, Oleaga A, Siles-Lucas M, Pérez-Sánchez R. Subolesin/akirin orthologs from Ornithodoros spp. soft ticks: cloning, RNAi gene silencing and protective effect of the recombinant proteins. Vet Parasitol. 2012;185(2-4):248-59.

6. Willadsen P, Riding GA, McKenna RV, Kemp DH, Tellam RL, Nielsen JN, Lahnstein J, Cobon GS, Gough JM. Immunologic control of a parasitic arthropod. Identification of a protective antigen from Boophilus microplus. J Immunol. 1989;143(4):1346-51.

7. Elvin $\mathrm{CM}$, Kemp DH. Generic approaches to obtaining efficacious antigens from vector arthropods. Int J Parasitol. 1994;24(1):67-79.

8. de la Fuente J, Moreno-Cid JA, Canales M, Villar M, de la Lastra JM, Kocan KM, Galindo RC, Almazán C, Blouin EF. Targeting arthropod subolesin/ akirin for the development of a universal vaccine for control of vector infestations and pathogen transmission. Vet Parasitol. 2011;181(1):17-22.

9. Allen JR, Humphreys SJ. Immunisation of guinea pigs and cattle against ticks. Nature. 1979:280(5722):491-3.

10. Rand KN, Moore T, Sriskantha A, Spring K, Tellam R, Willadsen P, Cobon GS. Cloning and expression of a protective antigen from the cattle tick Boophilus microplus. Proc Natl Acad Sci. 1989;86(24):9657-61.

11. Rodríguez M, Rubiera R, Penichet M, Montesinos R, Cremata J, Falcón V, Sánchez G, Bringas R, Cordovés C, Valdés M, Lleonart R. High level expression of the B. microplus Bm86 antigen in the yeast Pichia pastoris forming highly immunogenic particles for cattle. J Biotechnol. 1994;33(2):135-46.

12. Carreón D, de la Lastra JM, Almazán C, Canales M, Ruiz-Fons F, Boadella M, Moreno-Cid JA, Villar M, Gortázar C, Reglero M, Villarreal R. Vaccination with BM86, subolesin and akirin protective antigens for the control of tick infestations in white tailed deer and red deer. Vaccine. 2012:30(2):273-9.

13. de la Fuente J, Almazán C, Canales M, de la Lastra JM, Kocan KM, Willadsen P. A ten-year review of commercial vaccine performance for control of tick infestations on cattle. Anim Health Res Rev. 2007;8(1):23-8.

14. Canales M, Naranjo V, Almazán C, Molina R, Tsuruta SA, Szabó MP, Manzano-Roman R, de la Lastra JM, Kocan KM, Jiménez MI, Lucientes J. Conservation and immunogenicity of the mosquito ortholog of the tickprotective antigen, subolesin. Parasitol Res. 2009;105(1):97-111.

15. de la Fuente J, Kocan KM. Advances in the identification and characterization of protective antigens for recombinant vaccines against tick infestations. Expert Rev Vaccines. 2003;2(4):583-94.

16. Almazán C, Kocan KM, Bergman DK, Garcia-Garcia JC, Blouin EF, de la Fuente J. Identification of protective antigens for the control of Ixodes scapularis infestations using CDNA expression library immunization. Vaccine. 2003;21(13-14):1492-501.

17. Almazán C, Blas-Machado U, Kocan KM, Yoshioka JH, Blouin EF, Mangold AJ, de la Fuente J. Characterization of three Ixodes scapularis CDNAs protective against tick infestations. Vaccine. 2005;23(35):4403-16.

18. de la Fuente J, Almazán C, Blas-Machado U, Naranjo V, Mangold AJ, Blouin EF, Gortazar C, Kocan KM. The tick protective antigen, 4D8, is a conserved protein involved in modulation of tick blood ingestion and reproduction. Vaccine. 2006;24(19):4082-95.

19. Almazán C, Lagunes R, Villar M, Canales M, Rosario-Cruz R, Jongejan F, de la Fuente J. Identification and characterization of Rhipicephalus (Boophilus) microplus candidate protective antigens for the control of cattle tick infestations. Parasitol Res. 2010;106(2):471-9.

20. de la Fuente J, Manzano-Roman R, Naranjo V, Kocan KM, Zivkovic Z, Blouin EF, Canales M, Almazán C, Galindo RC, Step DL, Villar M. Identification of protective antigens by RNA interference for control of the lone star tick Amblyomma americanum. Vaccine. 2010;28(7):1786-95.

21. Goto A, Matsushita K, Gesellchen V, El Chamy L, Kuttenkeuler D, Takeuchi O, Hoffmann JA, Akira S, Boutros M, Reichhart JM. Akirins are highly conserved nuclear proteins required for NF-kB-dependent gene expression in Drosophila and mice. Nat Immunol. 2008;9(1):97-104.

22. Sutterwala FS, Flavell RA. Cascade into clarity. Nature. 2008:451(7176):254-5

23. Galindo RC, Doncel-Perez E, Zivkovic Z, Naranjo V, Gortazar C, Mangold AJ, Martín-Hernando MP, Kocan KM, de la Fuente J. Tick subolesin is an ortholog of the akirins described in insects and vertebrates. Dev Comp Immunol. 2009;33(4):612-7.
24. Hoffmann JA. The immune response of Drosophila. Nature. 2003;426(6962):33-8.

25. Artigas-Jerónimo S, Villar M, Cabezas-Cruz A, Valdés JJ, Estrada-Peña A, Alberdi P, De La Fuente J. Functional evolution of subolesin/akirin. Front Physiol. 2018;9:1612.

26. Artigas-Jerónimo S, Comín JJ, Villar M, Contreras M, Alberdi P, Viera IL, Soto L, Cordero R, Valdés JJ, Cabezas-Cruz A, Estrada-Peña A. A novel combined scientific and artistic approach for the advanced characterization of interactomes: The Akirin/Subolesin Model. Vaccines. 2020;8(1):77.

27. Debalke S, Habtewold T, Duchateau L, Christophides GK. The effect of silencing immunity related genes on longevity in a naturally occurring Anopheles arabiensis mosquito population from southwest Ethiopia. Parasit Vectors. 2019;12(1):174

28. Kumar S, Molina-Cruz A, Gupta L, Rodrigues J, Barillas-Mury C. A peroxidase/dual oxidase system modulates midgut epithelial immunity in Anopheles gambiae. Science. 2010;327(5973):1644-8.

29. Choe KM, Werner T, Stöven S, Hultmark D, Anderson KV. Requirement for a peptidoglycan recognition protein (PGRP) in Relish activation and antibacterial immune responses in Drosophila. Science. 2002;296(5566):359-62.

30. Gottar M, Gobert V, Michel T, Belvin M, Duyk G, Hoffmann JA, Ferrandon D, Royet J. The Drosophila immune response against Gram-negative bacteria is mediated by a peptidoglycan recognition protein. Nature. 2002:416(6881):640-4.

31. Ertürk-Hasdemir D, Broemer M, Leulier F, Lane WS, Paquette N, Hwang D, Kim CH, Stöven S, Meier P, Silverman N. Two roles for the Drosophila IKK complex in the activation of Relish and the induction of antimicrobial peptide genes. Proc Natl Acad Sci. 2009;106(24):9779-84.

32. Dushay MS, Asling B, Hultmark D. Origins of immunity: Relish, a compound Rel-like gene in the antibacterial defense of Drosophila. Proc Natl Acad Sci. 1996;93(19):10343-7.

33. Dong Y, Manfredini F, Dimopoulos G. Implication of the mosquito midgut microbiota in the defense against malaria parasites. PLoS Pathog. 2009;5:5.

34. Meister S, Agianian B, Turlure F, Relógio A, Morlais I, Kafatos FC, Christophides GK. Anopheles gambiae PGRPLC-mediated defense against bacteria modulates infections with malaria parasites. PLoS Pathog. 2009;5:8.

35. Bonnay F, Nguyen XH, Cohen-Berros E, Troxler L, Batsche E, Camonis J, Takeuchi O, Reichhart JM, Matt N. Akirin specifies NF-KB selectivity of Drosophila innate immune response via chromatin remodeling. EMBO J. 2014;33(20):2349-62.

36. Moreno-Cid JA, Jiménez M, Cornelie S, Molina R, Alarcón P, Lacroix MN, Pinal R, Delacour S, Lucientes J, Canales M, de la Lastra JM. Characterization of Aedes albopictus akirin for the control of mosquito and sand fly infestations. Vaccine. 2010;29(1):77-82.

37. da Costa M, Pinheiro-Silva R, Antunes S, Moreno-Cid JA, Custódio A, Villar $\mathrm{M}$, Silveira $\mathrm{H}$, de la Fuente J, Domingos A. Mosquito Akirin as a potential antigen for malaria control. Malar J. 2014;13(1):470.

38. Moreno-Cid JA, de la Lastra JM, Villar M, Jiménez M, Pinal R, Estrada-Peña A, Molina R, Lucientes J, Gortázar C, de la Fuente J, SUB/AKR Vaccine Study Group. Control of multiple arthropod vector infestations with subolesin/akirin vaccines. Vaccine. 2013;31(8):1187-96.

39. Dandalo LC, Brooke BD, Munhenga G, Lobb LN, Zikhali J, Ngxongo SP Zikhali PM, Msimang S, Wood OR, Mofokeng M, Misiani E. Population dynamics and Plasmodium falciparum (Haemosporida: Plasmodiidae) infectivity rates for the malaria vector Anopheles arabiensis (Diptera: Culicidae) at Mamfene, KwaZulu-Natal, South Africa. J Med Entomol. 2017;54(6):1758-66.

40. Letinić BD, Dahan-Moss Y, Koekemoer LL. Characterising the effect of Akirin knockdown on Anopheles arabiensis (Diptera: Culicidae) reproduction and survival, using RNA-mediated interference. PLOS ONE. 2020;15(2):e0228576.

41. Hargreaves K, Hunt RH, Brooke BD, Mthembu J, Weeto MM, Awolola TS, Coetzee M. Anopheles arabiensis and An. quadriannulatus resistance to DDT in South Africa. Medical and Veterinary Entomology. 2003;17(4):417-22.

42. Hunt RH, Brooke BD, Pillay C, Koekemoer LL, Coetzee M. Laboratory selection for and characteristics of pyrethroid resistance in the malaria vector Anopheles funestus. Med Vet Entomol. 2005;19(3):271-5.

43. Contreras M, Alberdi P, De Mera IG, Krull C, Nijhof A, Villar M, De La Fuente $J$. Vaccinomics approach to the identification of candidate protective 
antigens for the control of tick vector infestations and Anaplasma phagocytophilum infection. Front Cell Infect Microbiol. 2017;7:360.

44. Kitagawa K. Different effects of antagonists to histamine and serotonin receptors on itch-related behavior induced by substance $P$, histamine and serotonin in mice. Jpn J Pharmacol. 1997;73:93.

45. Hoff J. Methods of blood collection in the mouse. Lab Anim. 2000:29(10):47-53.

46. de la Fuente J, Moreno-Cid JA, Galindo RC, Almazán C, Kocan KM, Merino $\mathrm{O}$, de la Lastra JM, Estrada-Peña A, Blouin EF. Subolesin/Akirin vaccines for the control of arthropod vectors and vectorborne pathogens. Transbound Emerg Dis. 2013;60:172-8.

47. Nowak SJ, Aihara H, Gonzalez K, Nibu Y, Baylies MK. Akirin links twistregulated transcription with the Brahma chromatin remodeling complex during embryogenesis. PLoS Genet. 2012;8:3.

48. Ohm JR, Baldini F, Barreaux P, Lefevre T, Lynch PA, Suh E, Whitehead SA, Thomas MB. Rethinking the extrinsic incubation period of malaria parasites. Parasit Vectors. 2018;11(1):178.

49. Sinden RE. The biology of Plasmodium in the mosquito. Experientia. 1984;40(12):1330-43.

50. Aly AS, Vaughan AM, Kappe SH. Malaria parasite development in the mosquito and infection of the mammalian host. Annu Rev Microbiol. 2009:63:195-221.

51. Levashina EA. Immune responses in Anopheles gambiae. Insect Biochem Mol Biol. 2004;34(7):673-8.
52. Vlachou D, Schlegelmilch T, Runn E, Mendes A, Kafatos FC. The developmental migration of Plasmodium in mosquitoes. Curr Opin Genet Dev. 2006;16(4):384-91.

53. Suneja A, Gulia M, Gakhar SK. Blocking of malaria parasite development in mosquito and fecundity reduction by midgut antibodies in Anopheles stephensi (Diptera: Culicidae). Arch Insect Biochem Physiol. 2003;52(2):63-70.

54. Chugh M, AdakT, Sehrawat N, Gakhar SK. Effect of anti-mosquito midgut antibodies on development of malaria parasite, Plasmodium vivax and fecundity in vector mosquito Anopheles culicifacies (Diptera: Culicidae). Indian J Exp Biol. 2011;49(4):245-53.

55. Schetters T, Bishop R, Crampton M, Kopacek P, Lew-Tabor A, Maritz-Olivier C, Miller R, Mosqueda J, Patarroyo J, Rodriguez-Valle M, Scoles GA. Cattle tick vaccine researchers join forces in CATVAC. Parasit Vectors. 2016;9:105.

56. Contreras M, de la Fuente J. Control of Ixodes ricinus and Dermacentor reticulatus tick infestations in rabbits vaccinated with the Q38 Subolesin/ Akirin chimera. Vaccine. 2016;34(27):3010-3.

\section{Publisher's Note}

Springer Nature remains neutral with regard to jurisdictional claims in published maps and institutional affiliations.
Ready to submit your research? Choose BMC and benefit from:

- fast, convenient online submission

- thorough peer review by experienced researchers in your field

- rapid publication on acceptance

- support for research data, including large and complex data types

- gold Open Access which fosters wider collaboration and increased citations

- maximum visibility for your research: over $100 \mathrm{M}$ website views per year

At BMC, research is always in progress.

Learn more biomedcentral.com/submissions 